\title{
Heart failure disease management programs in Austria 2019
}

\section{A systematic survey of the Heart Failure Working Group and the Working Group for Cardiological Assistance and Care Personnel of the Austrian Society of Cardiology}

\author{
Gerhard Poelzl · Bettina Fetz - Johann Altenberger · Margret Fritsch • Johann Auer - Elfriede Stachl · \\ Armin Boehmer · Heinrich Frauendorfer · Christian Ebner · Friedrich Geyrhofer · Stefan Pötz · Annette Prim • \\ Hannes Alber · Renate de Grandis · Christiane Drack · Martin Hülsmann · Heart Failure Working Group and the \\ Working Group for Cardiological Assistance and Care Personnel of the Austrian Society for Cardiology
}

Received: 27 November 2019 / Accepted: 22 January 2020 / Published online: 18 February 2020

(C) The Author(s) 2020

Summary Heart failure (HF) is common and is associated with high morbidity, mortality and high health expenditure. A multidisciplinary disease management plan (DMP) can reduce morbidity and mortality, save costs and improve the quality of life. In Austria, three HF-specific DMPs are currently in a project phase and four established DMPs are active. Although programs are widely heterogeneous with respect to their intervention type, they pursue the same interventional goal by supporting seamless care between inpatient and community care settings with a multidisciplinary team. This survey presents a systematic survey of the HF-specific DMPs in Austria. Disparities between programs are highlighted and discussed. The

\section{All authors contributed equally to this survey on behalf of the Heart Failure Working Group and the Working Group for Cardiological Assistance and Care Personnel of the Austrian Society for Cardiology.}

Prof. G. Poelzl, MD, FESC, FHFA ( $₫)$

Department of Internal Medicine III, Cardiology \& Angiology, Medical University of Innsbruck, Anichstraße 35, 6020 Innsbruck, Tyrol, Austria gerhard.poelzl@tirol-kliniken.at

\section{B. Fetz, DGKP}

Landesinstitut für Integrierte Versorgung - LIV,

Innsbruck, Tyrol, Austria

\section{J. Altenberger, PD Dr}

Rehabilitation Center Grossgmain, Salzburg, Austria

\author{
M. Fritsch, DGKP \\ University Clinic of Internal Medicine II, Paracelsus Medical \\ University, Salzburg, Austria
}

J. Auer, Univ. Prof. Dr. · E. Stachl, DGKP

Department of Internal Medicine, St. Joseph's Hospital

Braunau, Braunau, Upper Austria, Austria nationwide establishment of HF-specific DMPs that integrate primary care and cardiology services including a regulation of the remuneration of stakeholders and program infrastructure is needed to decrease the burden of HF for both the individual and society.

Keywords Heart failure - Disease management plan · Multidisciplinary management

\section{Introduction}

Heart failure (HF) is a common chronic medical problem that is associated with considerable morbidity and mortality [1-3]. The prevalence of HF

A. Boehmer, Dr · H. Frauendorfer, DGKP

Department of Internal Medicine 1, Krems University Clinic, Krems, Lower Austria, Austria

C. Ebner, Dr · F. Geyrhofer, DGKP

Department of Internal Medicine 2, Hospital of the Order of St. Elizabeth, Linz, Upper Austria, Austria

S. Pötz, Dr · A. Prim, DGKP

Department of Internal Medicine, Hospital Hochsteiermark, Bruck/Mur, Styria, Austria

H. Alber, PD Dr · R. de Grandis, DGKP

Department of Internal Medicine \& Cardiology, Klinikum Klagenfurt am Wörthersee, Klagenfurt, Carinthia, Austria

C. Drack, DGKP

Rehabilitation Center Bad Ischl, Bad Ischl, Upper Austria, Austria

M. Hülsmann, PD Dr

University Clinic of Internal Medicine II, Medical University of Vienna, Vienna, Austria 
continuously rises due to changing demographics and better survival from cardiovascular diseases earlier in life. Meanwhile, there is abundant evidence to demonstrate the efficacy of a multidisciplinary team approach to meet the increasing complexity of $\mathrm{HF}$ management [3-7].

In 2017 the Heart Failure Working Group and the Working Group for Cardiological Assistance and Care Personnel of the Austrian Society of Cardiology published a position paper on the need for disease management programs (DMP) specific for heart failure in Austria [8]. That article defined essential elements of a DMP that were essentially based on recommendations made by the Heart Failure Association of the European Society of Cardiology (ESC) [3, 9]. The publication attracted much attention from healthcare providers and political representatives and enhanced an ongoing public debate about optimal management of chronic heart failure in Austria. Nevertheless, a comprehensive concept for nationwide coverage with heart failure-specific DMPs is still pending.

This article presents a systematic survey of currently active heart failure-specific DMPs in Austria. To ensure a high degree of clarity in presenting the heterogeneity of individual programs and allow for comparison, the summary is essentially based on the aforementioned position paper of the Austrian Society of Cardiology [8] and on a system of classification developed by The American Heart Association's Disease Management Taxonomy Writing Group [10]. This publication consciously refrains from presenting efficacy and cost effectiveness analyses since final or interim evaluation is not yet available for all programs. Final assessment of the programs will be addressed in a future publication.

Currently, seven individual DMPs are operating in six regions in Austria ranging from Carinthia (Klagenfurt) to Lower Austria (Krems), Salzburg, Styria, Tyrol, and Upper Austria (Braunau, Linz) (in alphabetical order). Kardiomobil Kärnten (January 2019-December 2020), Integrierte Versorgung von Patienten mit Herzinsuffizienz in Oberösterreich - IVH OÖ-Pilot (January 2017 to December 2019), and KardioStabil Braunau (June 2017 to unlimited) are still in a project phase, while Kardiomobil Salzburg (since 2008) [11], the Krems model (since 2008) [12] and HerzMobil Tirol (2012-2017 in a project phase, established since July 2017) [13-19] and HerzMobil Stmk (since January 2019) are already established programs.

A summary of heart failure-specific DMPs in Austria including the number of included patients as of May 2019, the proportion of women, and the drop-out rate are given in Table 1-4. Various aspects and disparities between programs are highlighted and discussed in detail. HerzMobil Tirol and HerzMobil Stmk as well as Kardiomobil Salzburg and Kardiomobil Kärnten are largely identical with a few exceptions and thus are mostly discussed as units.

\section{Model and form of intervention (Table 1)}

Most programs use hybrid tactics that combine either home visiting (HerzMobil Tirol/Stmk) or clinic-based (Krems Model, KardioStabil Braunau) approaches and telehealth care. Telehealth care includes regular telephone contacts with patients (Krems Model, KardioStabil Braunau) or mobile telephone-based home telemonitoring systems (HerzMobil Tirol/Stmk) [13]. HerzMobil Tirol also implements a broad network of resident physicians for home-based care delivery. Kardiomobil Salzburg/Kärnten is designed primarily as a nurse-based home visiting program and the IVH OÖ-Pilot is conceptionally a home and office-based program.

It is the primary goal of all programs to prevent readmissions among patients transitioning from hospitalization for acute heart failure (AHF) to outpatient healthcare facilities and to improve the quality of life (QoL). The transition is considered the most vulnerable period with the highest rates of readmission and mortality. In this sense all programs are designed as transitional care interventions.

\section{Patient population (Table 1)}

Most programs include patients who are hospitalized for AHF irrespective of the ejection fraction and the underlying cause and who are now transitioning to community care. The Krems Model restricts inclusion to patients with reduced ejection fraction. Additionally, outpatients with advanced HF (AdHF) are included in all programs except Kardiomobil Kärnten. Patients with serious or life-threatening comorbidities, dementia, need for permanent nursing home care, advanced frailty or communication problems, and lack of willingness to participate in a program are excluded. HerzMobil Tirol also explicitly excludes patients with a high degree of comorbid conditions (Charlson index >6) since experience in the early phase demonstrated that the program was unable to cope with this complexity.

\section{Intervention content (Table 1)}

Comprehensive patient education, monitoring of disease symptoms and optimization of treatment based on established HF guidelines are common to all programs.

A specialty-trained HF nurse meets with the patient while still in hospital (Krems Model, HerzMobil Tirol/Stmk, KardioStabil Braunau, IVH OÖ-Pilot) or at the latest within 2 weeks after discharge (Kardiomobil Salzburg/Kärnten) for extensive patient education including weight monitoring, medication adherence, symptom recognition, and diet. Patient education addresses patients either individually or as a group. In HerzMobil Tirol/Stmk patient education is supported by a patient brochure and also includes training in 


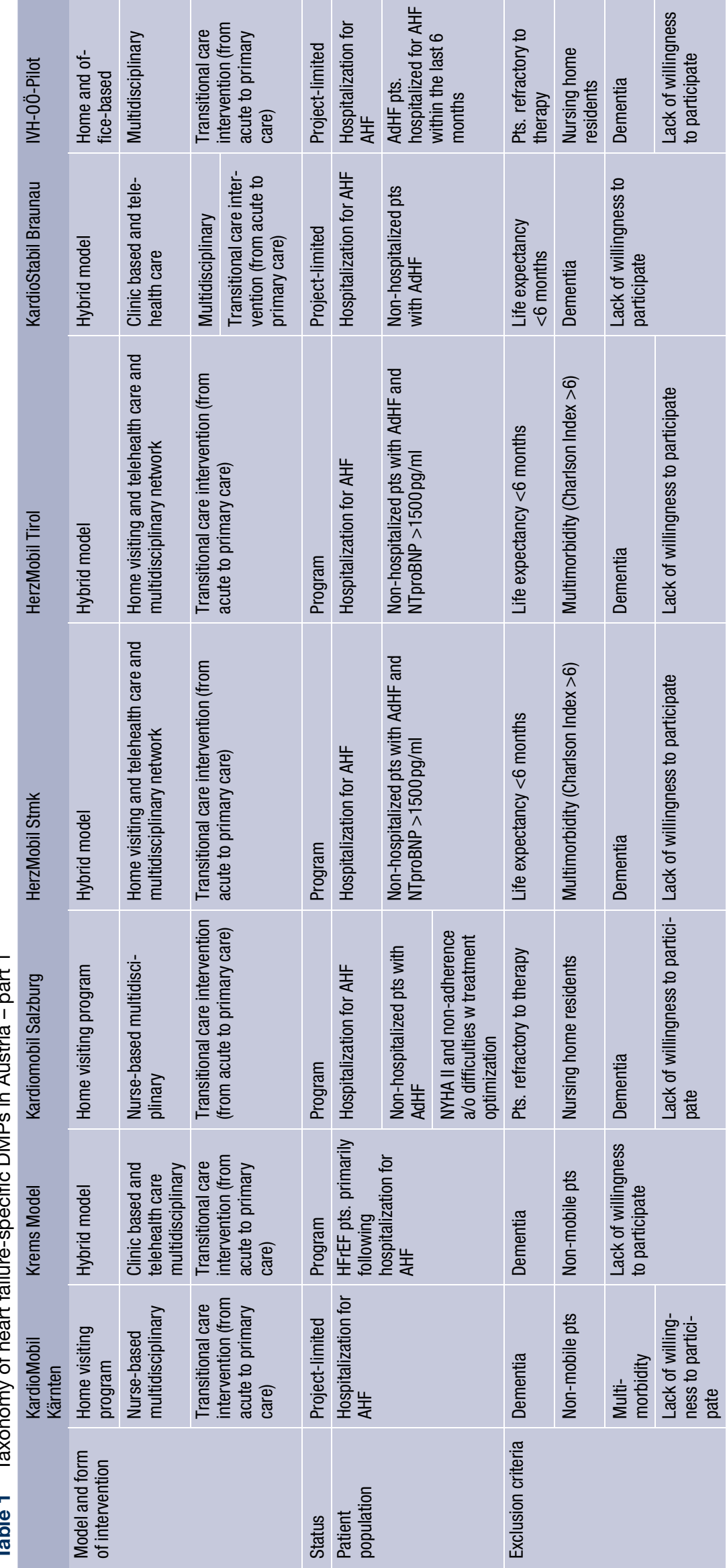




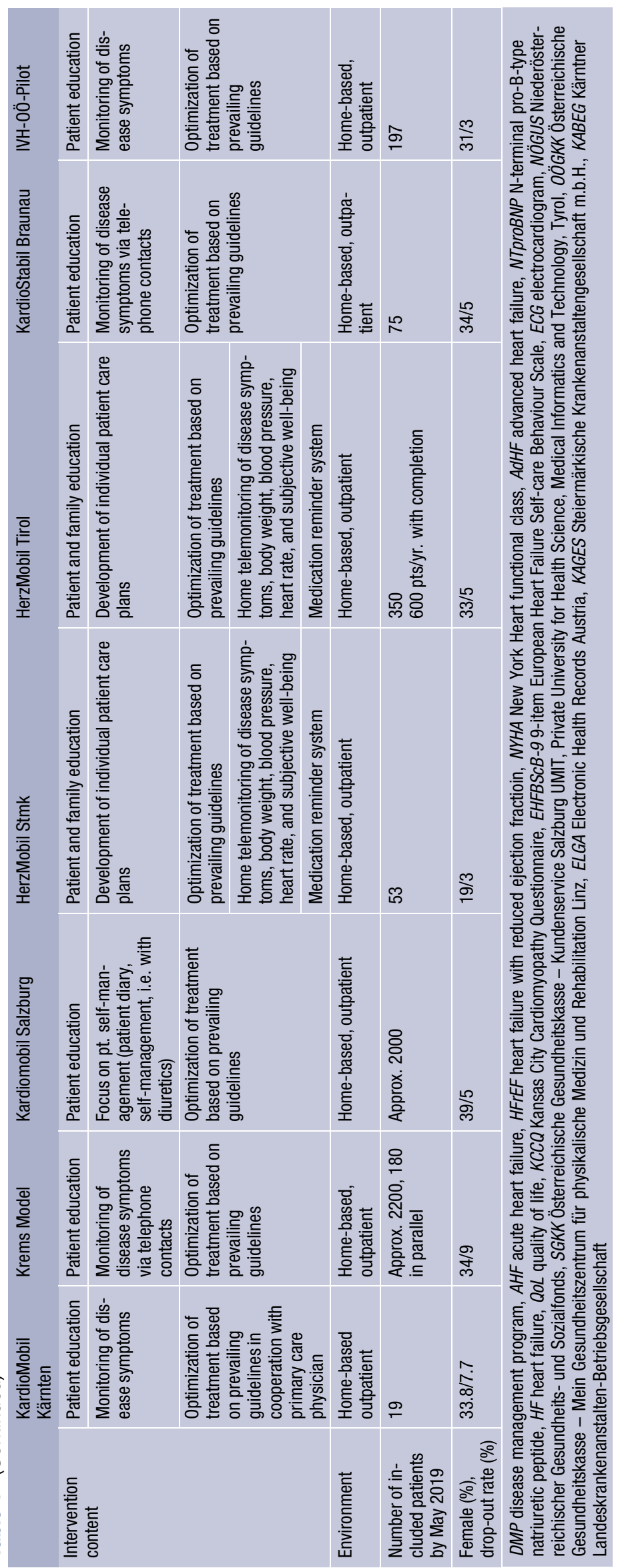


the handling of a provided mobile telephone, weight scale and blood pressure monitor. In both programs the nurse meets again with the patient within 1 week after discharge to repeat the training and ensure the availability of prescribed medication. The mobile telephone displays a constantly updated medication reminder on a daily basis over the course of the entire program.

In all programs an individual patient care plan is provided at hospital discharge and transmitted to the primary care physician. Individual treatment goals that include self-care behavior, medication adherence, QoL, physical activity, decongestion status and HF readmissions are set between the patients and the providers.

In the Krems Model and KardioStabil Braunau, patients' symptoms are monitored via office visits at the heart failure outpatient clinic and resident network physicians, respectively, in addition to regular telephone contacts for both programs. The INH OÖ-Pilot also provides regular office visits with resident network physicians and patient support from the local mobile support/social service.

In Kardiomobil Salzburg/Kärnten the HF nurse meets with the patient at home within 1-2 weeks after discharge at the discretion of the referring hospital or resident physician. At home visits special attention is paid to patient training involving family members and registration of the medication. Visits are conducted according to the Kardiomobil protocol and an electronic report is created and made available to the resident physician instantly via DaMe (A1, Vienna, Austria), the electronic health platform used statewide.

In HerzMobil Tirol the patient is assigned to a resident network physician and regular office visits are scheduled. In HerzMobil Tirol/Stmk physiologically relevant patient-associated parameters including heart rate, blood pressure, weight, well-being, and adherence to medication are obtained daily from patients via a remote electronic monitoring system.

\section{Delivery personnel (Table 2)}

\section{Nurses}

All programs are based on multidisciplinary teams that integrate specialty-trained nursing staffs. Nurses graduate from a comprehensive heart failure educational course at AZW Innsbruck (Training Center West for Allied Health Professions) that is certified by the ESC Heart Failure Association and the Austrian Society of Cardiology. Their duties include patient education and outpatient evaluation to relay clinically relevant findings to the patient's physician, thereby effecting more intensive management of the disease.

In the Krems Model and KardioStabil Braunau nursing staff are located in the hospital and patient contacts are made by telephone. In Kardiomobil
Salzburg/Kärnten and IVH OÖ-Pilot nurses personally meet with patients at home at various intervals according to the patients' needs. In IVH OÖ-Pilot nurses are supported by members of the local mobile support and/or social services, who underwent specific heart failure training.

In HerzMobil Tirol/Stmk nurses supervise equipment-obtained and remotely transmitted physiologic data plus information on subjective well-being and medication intake on a daily basis. Supervision is facilitated by an automatic event detection program that signals the need for therapeutic decisions triggered by missing values and off-limit measurements, where limits are individually defined. Alerts that cannot be handled by the nurse are forwarded to the network physician [13]. Also, nurses personally meet with patients at home within the first week after discharge for retraining in the presence of relatives and to check the availability of the prescribed medication. Another home visit is planned after 3 months for the purpose of assessing negotiated treatment goals. In the meantime, contacts via telephone and/or encrypted message service (EMS) and optional unplanned home visits are provided as needed.

\section{Physicians}

Hospital-based cardiologists or specialists for internal medicine are involved in all programs. It is their duty to include patients and design individualized treatment plans that are immediately transferred to all stakeholders. In the Krems Model and HerzMobil Stmk the patient is followed in an HF outpatient clinic on a regular basis. In HerzMobil Tirol/Stmk individual limits of physiologic data are also provided for the analyzing filter of the web-based HerzMobil telehealth software until necessary changes are made by the resident network physician. In all programs any serious decisions on patient management that become necessary during the program period are the responsibility of hospital-based cardiologists in the respective heart failure outpatient clinic and/or the medical program director. The IVH OÖ-Pilot provides a mandatory office visit in a heart failure outpatient clinic 1 year after inclusion and optional interim visits, if needed.

In KardioStabil Braunau, IVH OÖ-Pilot and HerzMobil Tirol a network of resident specialists for internal medicine and/or general practitioners is actively involved in patient management with particular attention focusing on the gradual optimization of HF medication. Network physicians are advised to follow a management plan delivered at discharge from hospital.

In Kardiomobil Salzburg/Kärnten the patient's resident physician (general practitioner or internist/ cardiologist) is the primary contact person for the heart failure nurse. Contacts mostly address optimization of heart failure medication and laboratory 


\begin{tabular}{|c|c|c|c|c|}
\hline 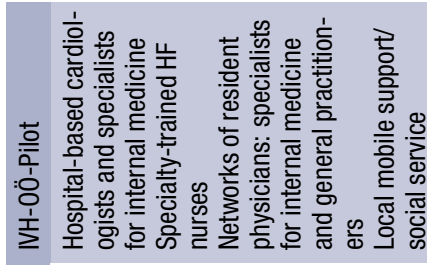 & 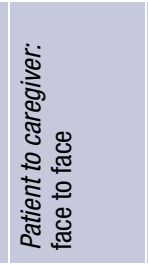 & 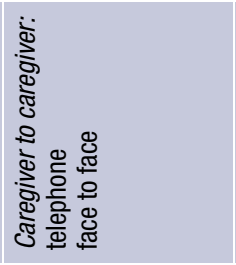 & 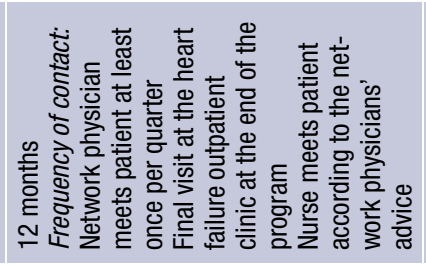 & 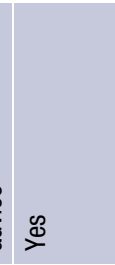 \\
\hline 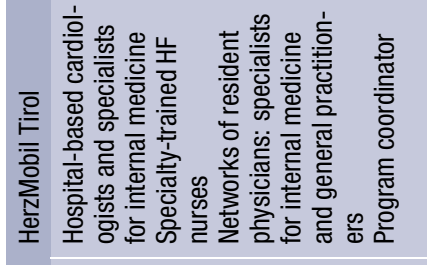 & 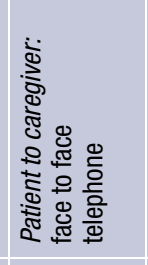 & 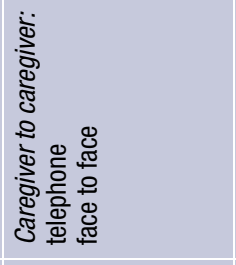 & 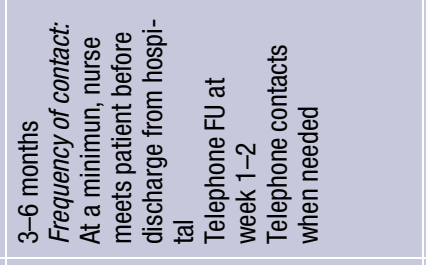 & $\stackrel{\mathscr{\infty}}{\rightleftharpoons}$ \\
\hline 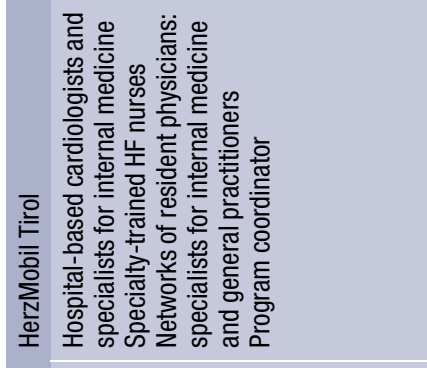 & 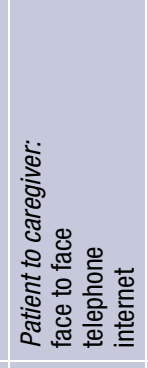 & 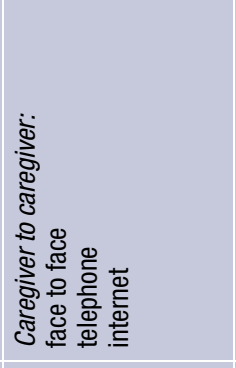 & 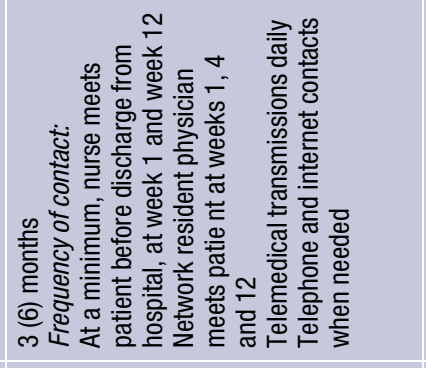 & $\stackrel{\mathscr{J}}{\nu}$ \\
\hline 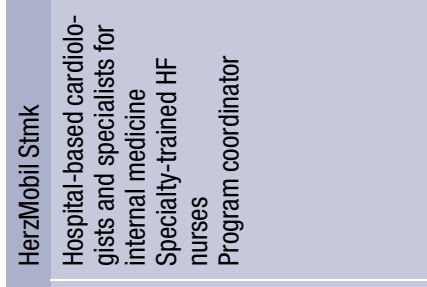 & 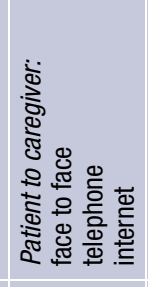 & 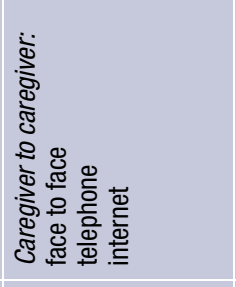 & 1 & $\stackrel{\mathscr{\infty}}{\rightleftharpoons}$ \\
\hline 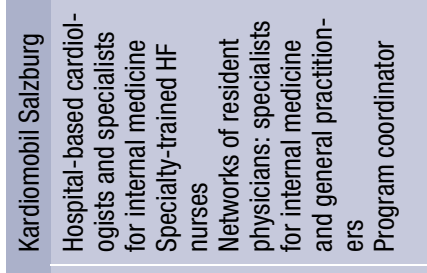 & 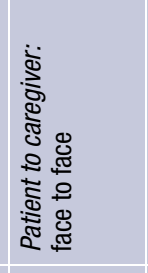 & 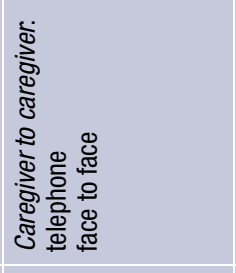 & 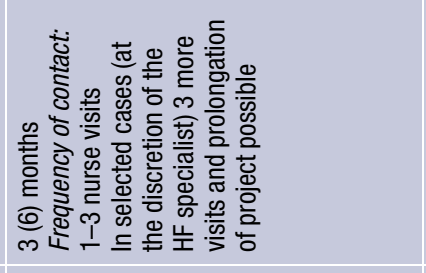 & $\stackrel{\mathscr{B}}{\rightleftharpoons}$ \\
\hline 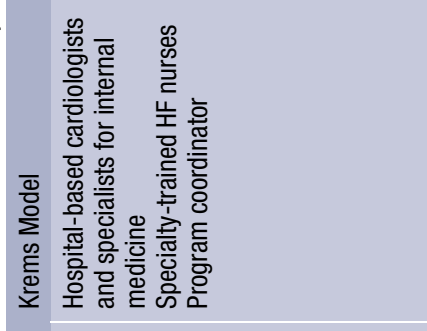 & 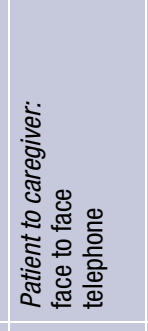 & 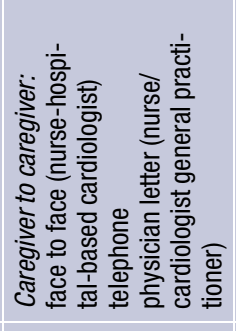 & 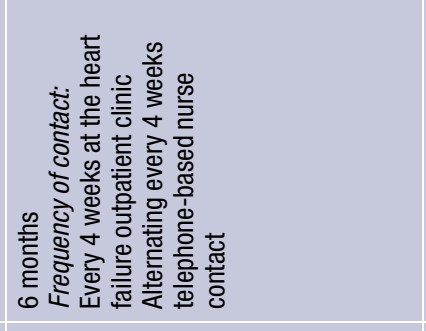 & $\stackrel{\infty}{\rightleftharpoons}$ \\
\hline 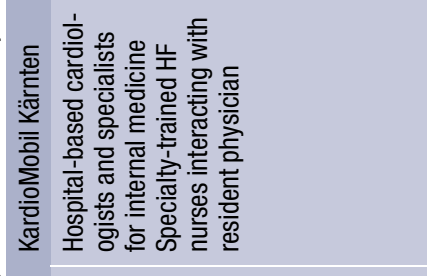 & 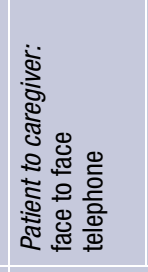 & 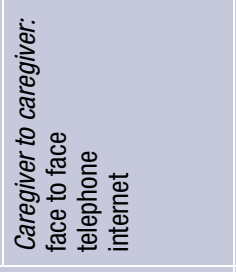 & 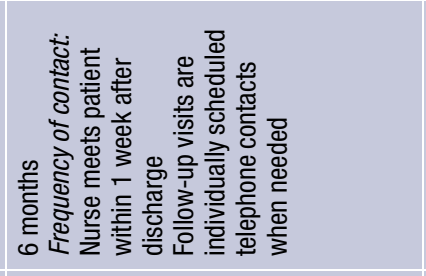 & $\stackrel{\mathscr{J}}{\rightleftharpoons}$ \\
\hline 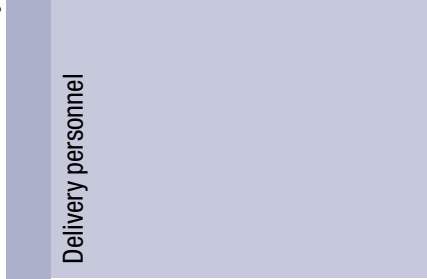 & 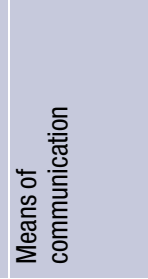 & & 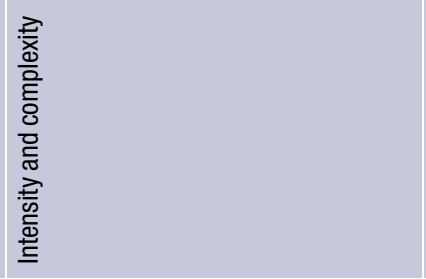 & 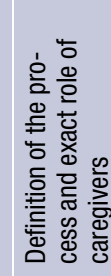 \\
\hline
\end{tabular}


controls. Nurses report on disease trajectory and relevant notes in the patient's medical diary. Hospitalbased cardiologists and the medical program director are available to the resident physician and the nurse when complex problems arise. In Kardiomobil Kärnten regular office visits in the heart failure outpatient clinic are planned after 6 and 12 months.

In HerzMobil Tirol an initial consultation with the resident network physician is scheduled within the first week after discharge and two more visits at weeks 4 and 12. Consultations include laboratory analysis with special attention to renal function and electrolytes. It is the responsibility of the network physicians to optimize HF treatment based on established HF guidelines and to react in a timely manner in the case of disease deterioration and thus prevent rehospitalization. Network physicians review the summarized transmitted data from assigned patients on a weekly basis or immediately in the case of upgraded alerts. Necessary therapy adjustments according to predefined algorithms are transferred to patients either by telephone, the nurse or as part of an additional office visit to ascertain timely interventions [13].

\section{Coordinator}

A medical coordinator orchestrates all stakeholders and manages efficient cooperation of the involved partners. This is a full-time position in HerzMobil Tirol held by a heart failure nurse and a part-time position staffed by either a nurse or a hospital-based physician in all other programs.

Kardiomobil Salzburg is coordinated by a non-medical staff member of the organizational platform AVOS.

\section{Means of communication (Table 2)}

Augmented face to face interaction is the means of communication between care providers and patients in all programs. Telephone contact for the purpose of monitoring clinical symptoms, reinforcing educational content, offering encouragement, and responding to patient questions is implemented in all programs except Kardiomobil Kärnten and IVH OÖ-Pilot. HerzMobil Tirol/Stmk also uses a remote electronic monitoring system (HerzMobil telehealth system) that works with near field communication (NFC)-enabled mobile telephones and Bluetooth low energy (BLE)enabled medical devices to record measurements of patients' weight, blood pressure, heart rate, and information on subjective well-being and medication intake. Encrypted message service (EMS) via mobile telephone is also used to exchange messages between patients and caregivers [14, 15].

In all programs communication among caregivers, which is essential for interaction between program elements, is conducted face to face and via telephone. In HerzMobil Tirol/Stmk information is also shared instantly on the web-based HerzMobil telehealth system.

In Kardiomobil Salzburg information is stored on the web-based Kardiomobil software and shared with resident physicians via DaMe.

\section{Intensity and complexity (Table 2)}

\section{Duration}

Duration of the provided structured intervention ranges from 3 (KardioStabil Braunau, HerzMobil Tirol/ Stmk, Kardiomobil Salzburg) to 6 (Kardiomobil Kärnten, Krems Model) or 12 (IVH OÖ-Pilot) months. KardioStabil Braunau, HerzMobil Tirol/Stmk and Kardiomobil Salzburg allow a one-time extension to 6 months if negotiated treatment goals are not achieved after 3 months.

\section{Frequency/periodicity}

In HerzMobil Tirol/Stmk three regular face-to-face meetings with the nurse (one in-hospital, one home visit within 1 week after discharge and another one at the end of the program) and three regular office meetings with the network physician are scheduled. Event-related telephone/EMS contacts are intended. If necessary, additional home or office visits are possible.

In KardioMobil Salzburg regular home visits with the nurse are planned for 1-3 dates within 3 months. Another 1-3 visits are possible at the discretion of the resident physician or the medical program director. In IVH OÖ-Pilot an office visit with the resident network physician is intended at least once per quarter year. Home visits by the nurse are necessary in about $15 \%$ of included patients. In the Krems Model an office visit in the heart failure outpatient clinic is scheduled every month, alternating with phone contacts made by the nurse.

\section{Complexity}

All programs offer a mix of various individual components resulting in intermediate to high complexity. Individualized discharge planning and extensive selfmanagement education are common to all programs.

The Krems Model and KardioStabil Braunau involve one hospital each. Patient education is provided still during hospitalization and disease symptoms are monitored mostly by telephone contacts. Optimization and adjustment of medication is managed at prescheduled visits at the heart failure outpatient clinic (Krems Model) or at prescheduled office visits with the resident network physician (KardioStabil Braunau).

KardioMobil Salzburg includes eight hospital and two cardiac rehabilitation centers throughout the state. Patient education and monitoring of disease 
symptoms are managed by a mobile nurse during home visits. Treatment optimization is stipulated in the individual patient care plan and executed by the resident physician. The heart failure nurse monitors the work off the care plan and the patient's adherence to the medication plan. This is a key element in the electronic protocol and is reported to the resident physician. Disease monitoring is based on patient self-monitoring (blood pressure, heart rate, weight) and is recorded in a medical diary. The system implements six nurses, who are assigned to dedicated geographic regions and who collaborate with the regional hospitals. Each nurse is in regular contact with the regional hospital-based cardiologists/specialists for internal medicine and also offers in-hospital education, if requested. Organization and execution of nurse visits as well as data management are processed via the Kardiomobil software system.

The IVH OÖ-Pilot includes four hospitals. Patient education is managed by a cardiologist and a $\mathrm{HF}$ nurse while patients are still in hospital. Monitoring of symptoms and constant advising of patients are handled by the local support and social service. Treatment optimization is handled by the resident network physician or the heart failure outpatient clinic.

HerzMobil Tirol/Stmk currently involve seven and two hospitals, respectively. Patient education is provided during hospitalization and during home visits. Disease monitoring is managed via telemonitoring, where telemedical data are reviewed daily by a nurse and weekly by a resident network physician (HerzMobil Tirol) and a hospital physician (HerzMobil Stmk). The complexity of these programs arises from a network of HF nurses and resident network physicians, who communicate via an internet-based platform (HerzMobil telehealth system) operated in the local eHealth infrastructure of Tirol/Stmk. Resident network physicians define and implement treatment optimization at prescheduled office visits or else by timely reacting to alerts provided by the telemonitoring system.

\section{Monitoring of program outcomes/outcome measures (Table 3)}

Monitoring of outcomes is an integral element of all programs. Assessment of negotiated treatment goals is common to all programs. Hospital readmissions and mortality at the end of the program and at 12 months are registered in most but not all programs. Some programs also control for QoL, laboratories including NTproBNP, and echocardiography at various time points. Details are shown in Table 1. Additionally, quality of HF medication and adherence to medication are recorded in HerzMobil Tirol/Stmk [17] and KardioMobil Kärnten.

\section{Quality control (Table 3)}

Quality assurance (QA) and quality control (QC) are integral to all programs, although with some inconsistencies. Common to all programs is the QA warranty by providing an exact definition of the respective process flow and the roles of involved caregivers, the majority of which are specialty-trained. All programs offer regular advanced retraining for physicians and nurses. An introductory workshop is mandatory for first-time stakeholders and regular quality circles and case conferences are provided in most programs. Quality circles are used to implement necessary optimization steps and restructuring of the respective program. QC is based on benchmark evaluation, where assessed parameters differ between programs (Table 1). Heart failure rehospitalizations and mortality are assessed in all but two programs (Krems Model, HerzMobil Stmk.) and can be used for future cost-effectiveness analysis. Optimization of heart failure therapy and patient adherence to medication is controlled for in HerzMobil Tirol and KardioMobil Kärnten. Kardiomobil Salzburg is required to submit an annual performance report to the funding institution. Two programs are subjected to an annual external quality control by the provider (Krems Model) and the Private University for Health Science, Medical Informatics and Technology, Tyrol (HerzMobil Tirol) [19].

\section{Algorithm for troubleshooting (Table 3)}

Timely reaction to clinical deterioration of involved patients is ensured differently. In all programs the nurse who monitors disease symptoms by phone or face-to-face contacts, alerts the network physician, if needed. In Kardiomobil Salzburg the patient's resident physician is informed first. In the case of serious problems the alert is upgraded to the hospitalbased network physician. In KardioStabil Braunau the hospital-based network physician is the primary contact person, IVH OÖ-Pilot and Kardiomobil Kärnten provide a $24 / 7$ telephone hotline that is handled by a hospital-based cardiologist.

In HerzMobil Tirol/Stmk the nurse responds to an automatic event detection program that signals the need for therapeutic actions based on patient notes and/or missing or off-limit measurements, where limits are individually defined by the network physician. Alerts that cannot be handled by the nurse are upgraded to the network physician. In both programs predefined algorithms propose the reaction in cases of patient counselling and adjustment of HF medication.

\section{Transition to regular care (Table 3)}

Transition of patients from DMP to regular community care at the end of the program is supported by 


\begin{tabular}{|c|c|c|c|}
\hline 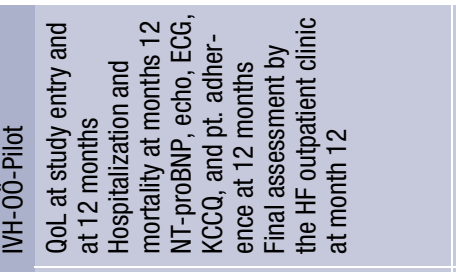 & 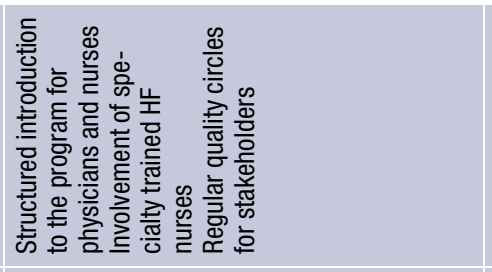 & 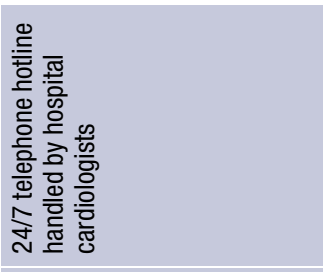 & 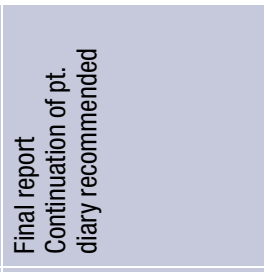 \\
\hline 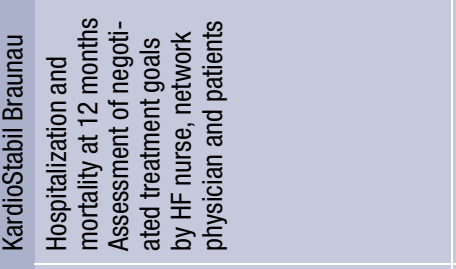 & 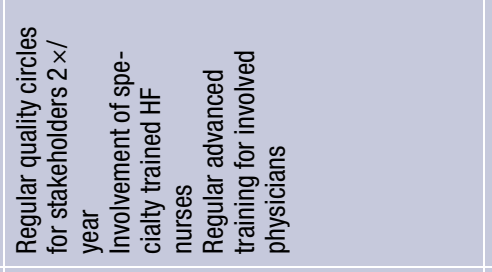 & 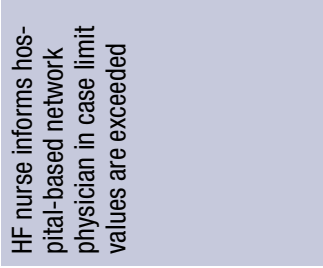 & 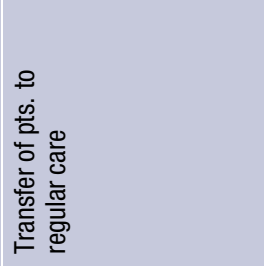 \\
\hline 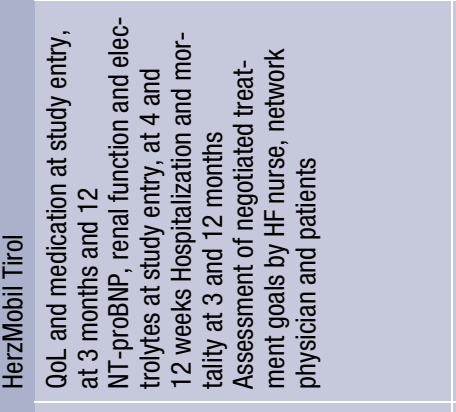 & 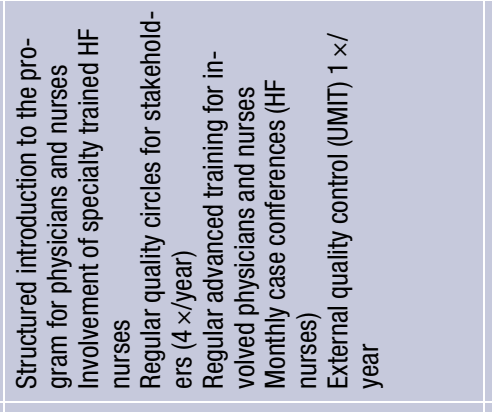 & 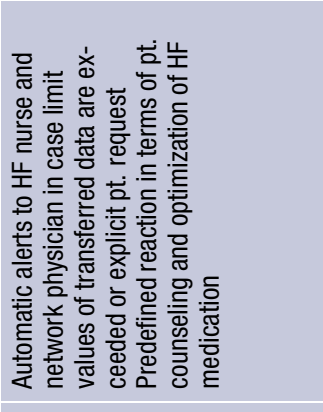 & 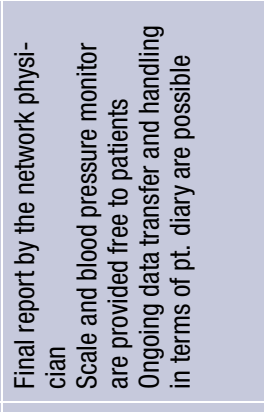 \\
\hline 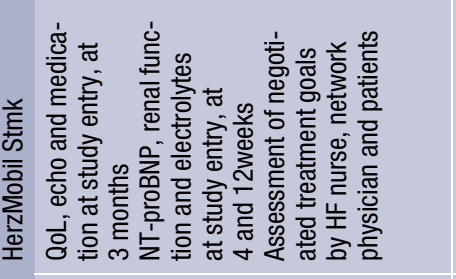 & 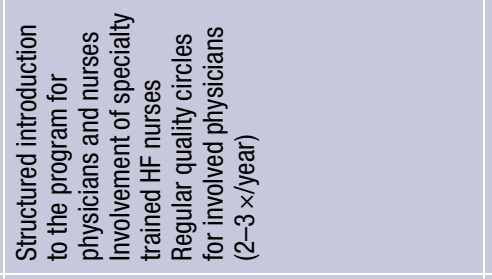 & 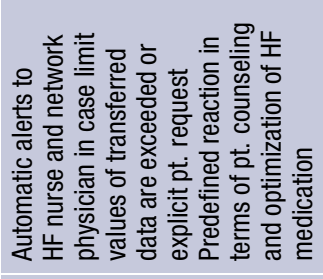 & 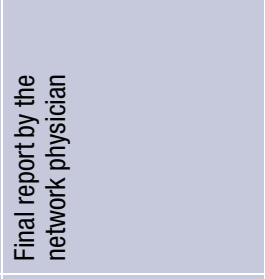 \\
\hline 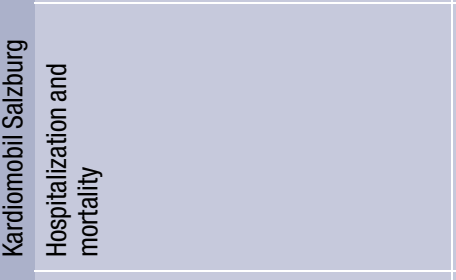 & 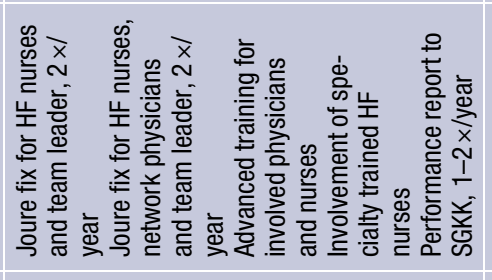 & 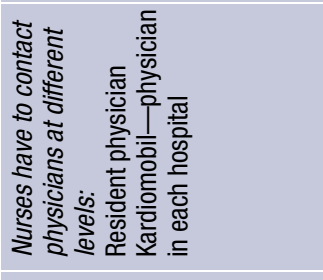 & 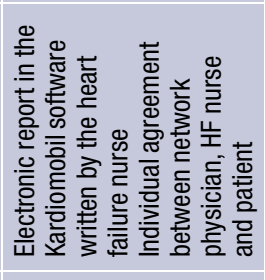 \\
\hline 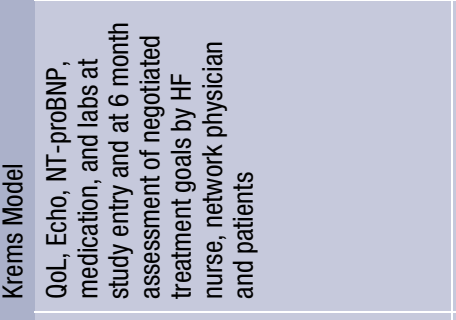 & 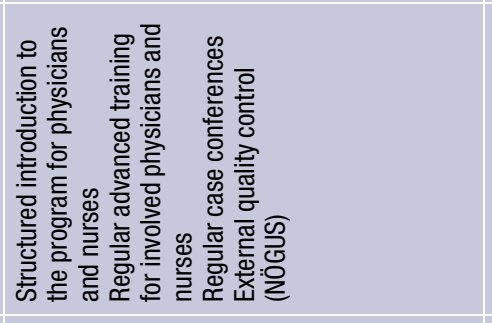 & 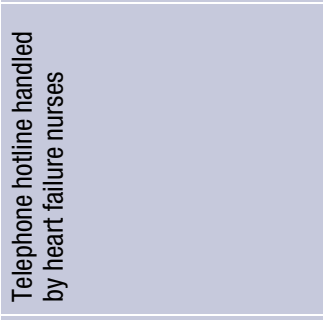 & 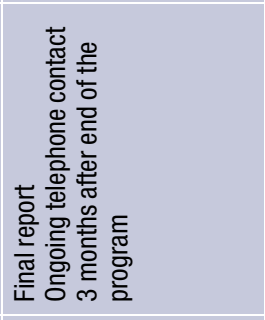 \\
\hline 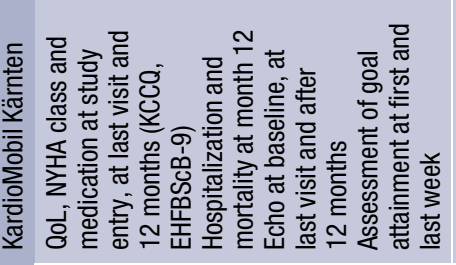 & 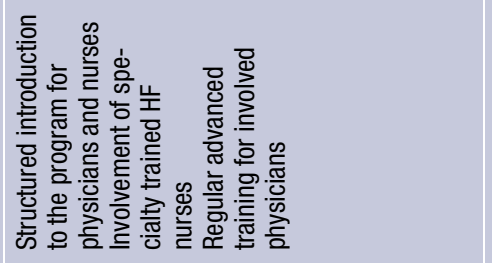 & 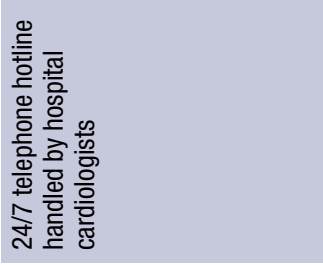 & 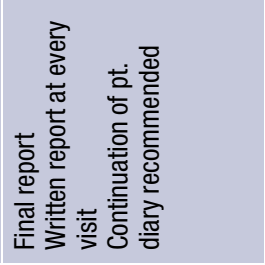 \\
\hline 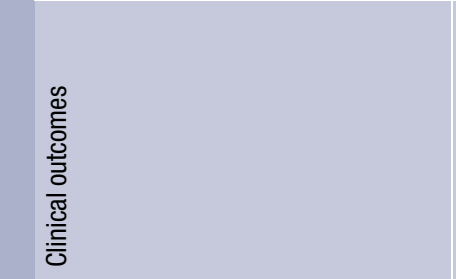 & 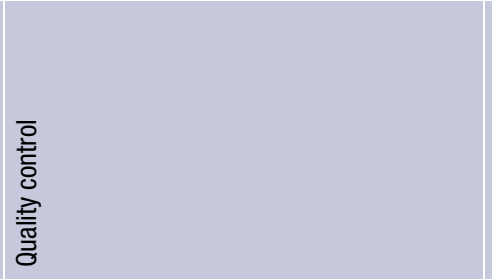 & 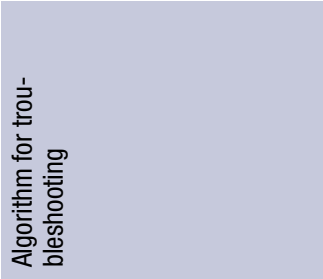 & 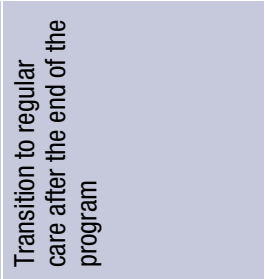 \\
\hline
\end{tabular}


a patient report. The Krems Model offers ongoing telephone contact with the nurse for an additional 3 months. HerzMobil Tirol gives the patients a free scale, blood pressure monitor and mobile telephone and offers ongoing data transfer and handling in terms of a patient diary.

\section{Provider and incentives (Table 4)}

KardioStabil Braunau and the Krems Model are provided by the local hospital operator (KH St. Josef Braunau, Niederösterreichischer Gesundheits- u. Sozialfonds - NÖGUS). Kardiomobil Kärnten, IVH OÖ-Pilot and HerzMobil Stmk are provided by the local hospital operator (Kärntner Krankenanstalten-Betriebsgesellschaft - KABEG, Oö Gesundheitsholding GmbH - GESPAG/Ordensklinikum Linz GmbH/JKU Linz, Steiermärkische Krankenanstaltgesellschaft - KAGes) commissioned and authorized by the healthcare fund of the respective state. In HerzMobil Tirol, operational matters are outsourced by the healthcare fund of the State of Tyrol to Landesinstitut für integrierte Versorgung - LIV. Kardiomobil Salzburg is outsourced and operated by Arbeitskreis für Vorsorgemedizin AVOS.

Temporary limited project funding for KardioStabil Braunau is provided by the hospital operator, for Kardiomobil Kärnten and IVH OÖ-Pilot by the Health Care Fund of the particular state. Temporary unlimited funding for the Krems Model is warranted by the hospital operator and for Kardiomobil Salzburg by the Health Care Fund of the State of Salzburg and the health insurance carriers of the state of Salzburg. In HerzMobil Tirol/Stmk the program infrastructure is provided by the Health Care Fund of the particular state. Full-time employment of nurses is funded by a 50:50 agreement between the Health Care Fund of the particular state and the compulsory health insurance carriers. Network physicians are remunerated at a flat rate per patient that is paid by the compulsory health insurance carriers.

\section{Future directions (Table 4)}

All programs that are still in a project phase are pursuing final evaluation and definite establishment of the program. Kardiomobil Salzburg is aspiring to implement a telemedical component and set up a defined network of resident physicians. HerzMobil Tirol/Stmk are striving to integrate all hospitals in the respective state and to connect the program to the national electronic health record-ELGA. HerzMobil Tirol is in the process of testing the implementation of a cardiac rehabilitation element into the program.

\section{Conclusion}

Prevalence and cost of care for HF will continue to increase because of the aging of the population. Ef- ficacy has been demonstrated for a multidisciplinary team approach to meet the increasing complexity of HF management [3, 4, 6, 7, 9].

In Austria seven HF-specific DMPs are currently active. Programs are widely heterogeneous with respect to intervention type. This is mostly due to the geographic and political diversity and to differences in the local health infrastructure between Austria's states. Also, geographical conditions in terms of rural-urban distribution and also budgetary requirements substantially impact the respective intervention type. Still, they pursue the same intervention goal by supporting seamless care between inpatient and community care settings in a multidisciplinary team. Programs are either hospital-based or home-based and consistently integrate specialty-trained nursing staff. Depending on the mix of individual components, complexity ranges from regular telephone contacts and/or home visits to mobile telephonebased remote telemonitoring systems involving networks of resident physicians. While three programs are still in a project phase, four programs are already well established. In two programs the remuneration of stakeholders is clearly set down in contracts with compulsory health insurance companies.

Inclusion criteria are defined with some blurs in all programs. Patients with multimorbidities and in the end-of-life period are currently not included in any of the programs. Care for these patients exceeds the capacity and financial restrictions of programs in this early phase but is a worthwhile goal for the future. Quality assurance is guaranteed in all programs and measures for quality control are implemented.

Finally, it needs to be noted that despite the fact that it is very gratifying that in Austria some DMPs for heart failure are already running, this is still only a first step in the right direction. The ultimate goal must be the nationwide establishment of HF-specific DMPs that integrate primary care with cardiology services including clear-cut regulation of the remuneration of stakeholders and program infrastructure. This is the most likely way to diminish the burden of HF for both the individual and society. It is our hope that presentation of already existing heart failure-specific DMPs will stimulate and encourage the stepwise institution of similar facilities in all Austrian states in order to meet local needs and requirements.

Funding Open access funding provided by University of Innsbruck and Medical University of Innsbruck.

\section{Compliance with ethical guidelines}

Conflict of interest G. Poelzl, B. Fetz, J. Altenberger, M. Fritsch, J. Auer, E. Stachl, A. Boehmer, H. Frauendorfer, C. Ebner, F. Geyrhofer, S. Pötz, A. Prim, H. Alber, R. de Grandis, C. Drack, and M. Hülsmann declare that they have no competing interests. 


\section{review article}

\begin{tabular}{|c|c|c|c|c|c|}
\hline $\begin{array}{l}\text { to } \\
\bar{n} \\
: 0 \\
0 \\
1 \\
\underline{1} \\
\underline{1}\end{array}$ & 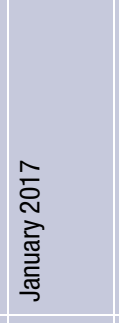 & 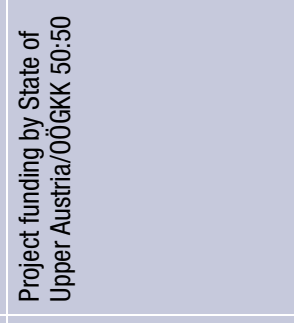 & 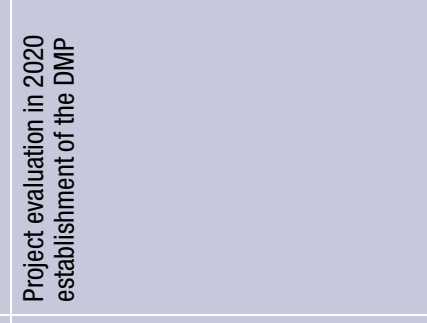 & 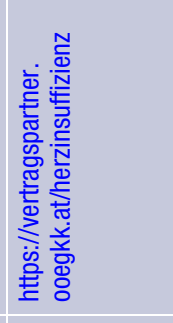 & 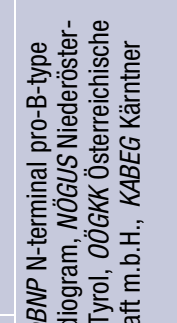 \\
\hline 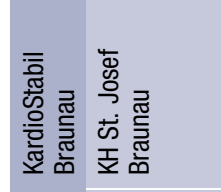 & 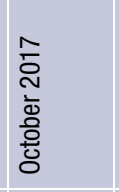 & 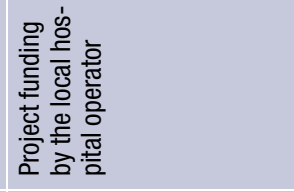 & 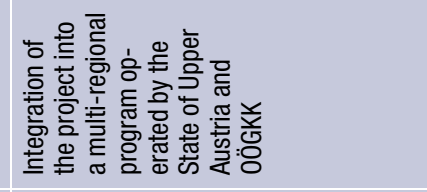 & 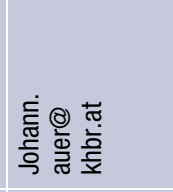 & 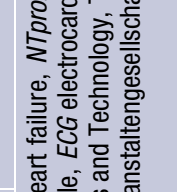 \\
\hline 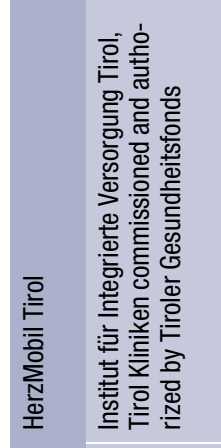 & 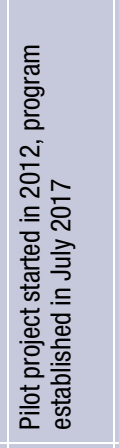 & 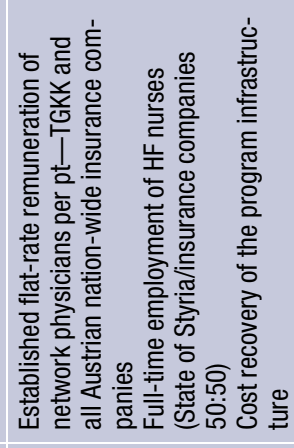 & 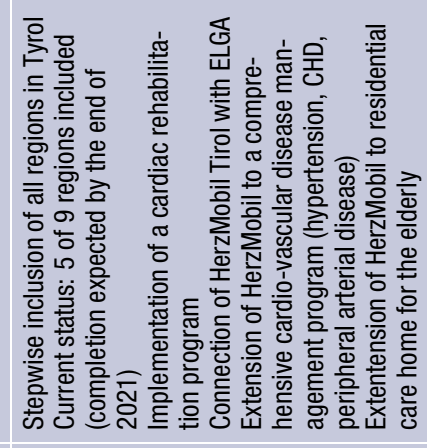 & D. & 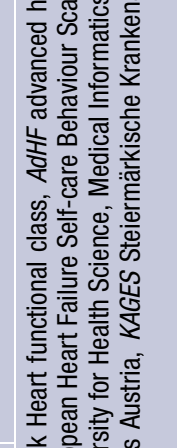 \\
\hline 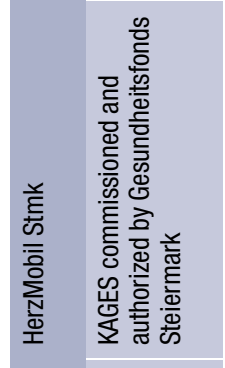 & 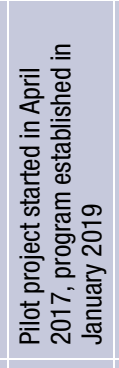 & 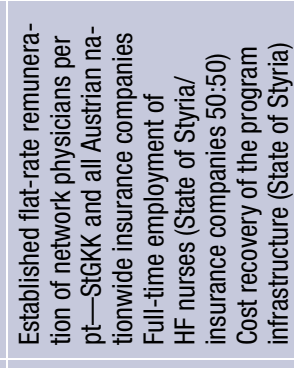 & 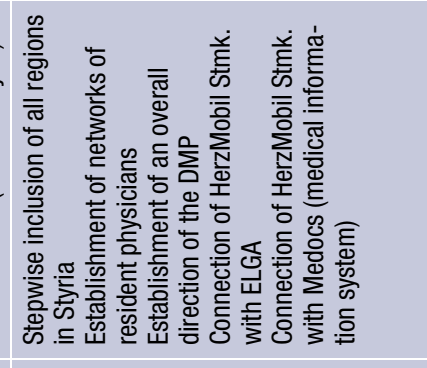 & 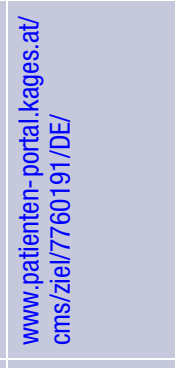 & 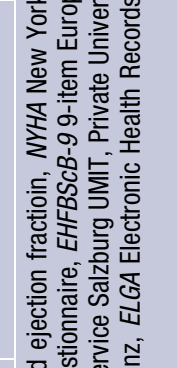 \\
\hline 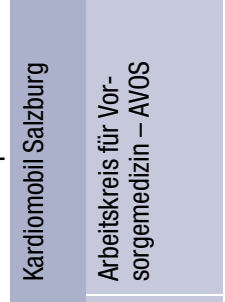 & 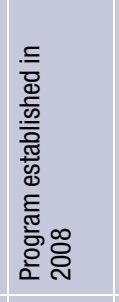 & 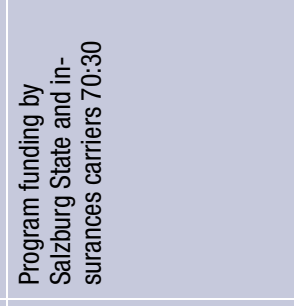 & 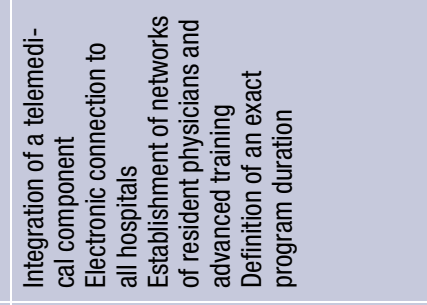 & 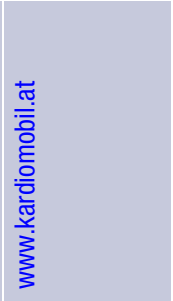 & 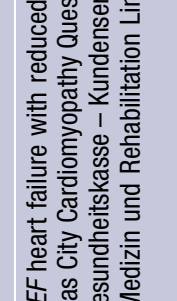 \\
\hline 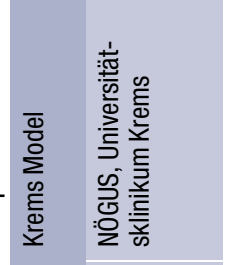 & 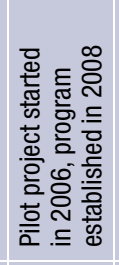 & 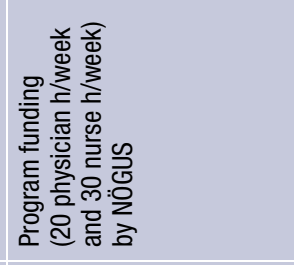 & 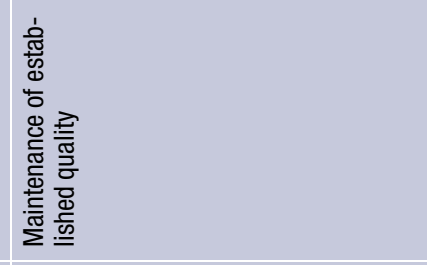 & 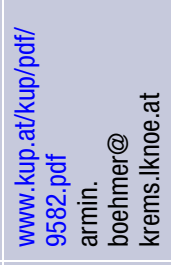 & 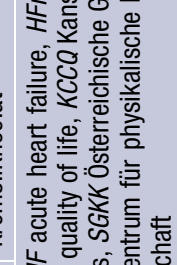 \\
\hline 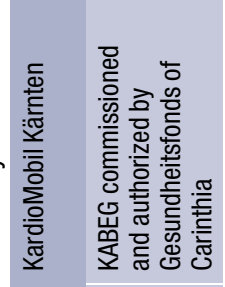 & 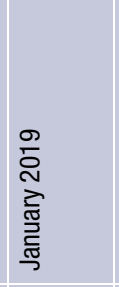 & 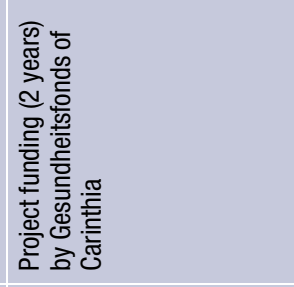 & 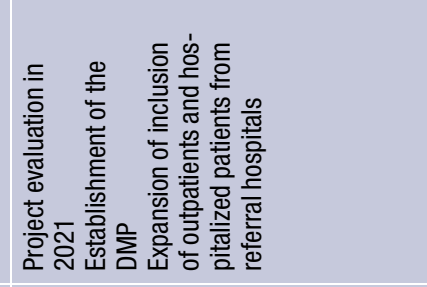 & 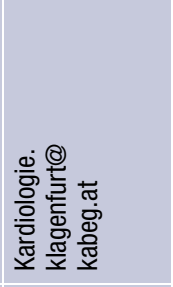 & 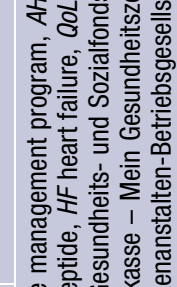 \\
\hline 离 & 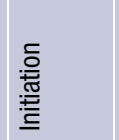 & 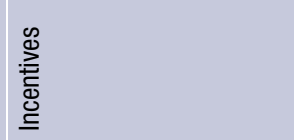 & 造 & 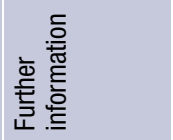 & 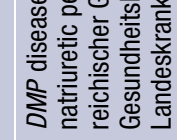 \\
\hline
\end{tabular}


Open Access This article is licensed under a Creative Commons Attribution 4.0 International License, which permits use, sharing, adaptation, distribution and reproduction in any medium or format, as long as you give appropriate credit to the original author(s) and the source, provide a link to the Creative Commons licence, and indicate if changes were made. The images or other third party material in this article are included in the article's Creative Commons licence, unless indicated otherwise in a credit line to the material. If material is not included in the article's Creative Commons licence and your intended use is not permitted by statutory regulation or exceeds the permitted use, you will need to obtain permission directly from the copyright holder. To view a copy of this licence, visit http://creativecommons.org/licenses/by/4.0/.

\section{References}

1. Levy D, Kenchaiah S, Larson MG, et al. Long-term trends in the incidence of and survival with heart failure. N Engl J Med. 2002;347(18):1397-402.

2. Mosterd A, Hoes AW. Clinical epidemiology of heart failure. Heart. 2007;93(9):1137-46.

3. Ponikowski P, Voors AA, Anker SD, et al. 2016ESC Guidelines for the diagnosis and treatment of acute and chronic heart failure: the Task Force for the diagnosis and treatment of acute and chronic heart failure of the European Society of Cardiology (ESC)Developed with the special contribution of the HeartFailureAssociation (HFA) of theESC. Eur HeartJ. 2016;37(27):2129-200.

4. Koehler F, Koehler K, Deckwart O, et al. Efficacy of telemedical interventional management in patients with heart failure (TIM-HF2): a randomised, controlled, parallel-group, unmasked trial. Lancet. 2018;392(10152):1047-57.

5. Davidson PM, Newton PJ, Tankumpuan T, et al. Multidisciplinary management of chronic heart failure: principles and future trends. Clin Ther. 2015;37(10):2225-33.

6. Berger R, Moertl D, Peter S, et al. N-terminal pro-B-type natriuretic peptide-guided, intensive patient management in addition to multidisciplinary care in chronic heart failure a 3-arm, prospective, randomized pilot study. J Am Coll Cardiol. 2010;55(7):645-53.

7. Adlbrecht C, Huelsmann M, Berger R, et al. Cost analysis and cost-effectiveness of NT-proBNP-guided heart failure specialist care in addition to home-based nurse care. Eur J Clin Invest. 2011;41(3):315-22.

8. MoertlD, Altenberger J, Bauer N, etal. Disease management programs in chronic heart failure: position statement of the Heart Failure Working Group and the Working Group of the Cardiological Assistance and Care Personnel of the Austrian
Society of Cardiology. Wien Klin Wochenschr. 2017;129(2324):869-78.

9. McDonagh TA, Blue L, Clark AL, et al. European society of cardiology heartfailure association standardsfor delivering heart failure care. Eur JHeart Fail. 2011;13(3):235-41.

10. Krumholz HM, Currie PM, Riegel B, et al. A taxonomy for disease management: a scientific statementfrom theAmerican Heart Association Disease Management Taxonomy Writing Group. Circulation. 2006;114(13):1432-45.

11. Altenberger JSR, Eichinger J, Matzinger M, et al. Impact of a nurse-led-home-based care program (Kardiomobil) on mortality and rehospitalizations in chronic heart failure (CHF) patients. Eur Heart J.2010;31:903.

12. Böhmer A, Wieser M, Weywar I, Frauendorfer H, Kronik G. Das „Kremser Modell“: Erfolgreiches Disease-Management zur Betreuung von Patienten mit Herzinsuffizienz. JKardiol. 2011;18:299-306.

13. Von der Heidt A, Ammenwerth E, Bauer K, et al. HerzMobil Tirol network: rationale for and design of a collaborative heartfailure disease management program in Austria. Wien Klin Wochenschr. 2014;126(21-22):734-41.

14. Modre-Osprian R, Polzl G, Von Der Heidt A, et al. Closedloop healthcare monitoring in a collaborative heart failure network. Stud Health Technol Inform. 2014;198:17-24.

15. Modre-Osprian R, Gruber K, Kreiner K, et al. Textual analysis of collaboration notes of the telemedical heart failure network HerzMobil Tirol. Stud Health Technol Inform. 2015;212:57-64.

16. Ammenwerth E, Woess S, Baumgartner C, et al. Evaluation of an integrated telemonitoring surveillance system in patients with coronary heart disease. Methods Inf Med. 2015;54(5):388-97.

17. Kreiner K, Welte S, Modre-Osprian R, et al. A personalized feedback system for supporting behavior change for patients after an acute myocardial infarction. Stud Health Technol Inform. 2015;212:50-6.

18. Eggerth A, Modre-Osprian R, Hayn D, et al. Comparison of body weight trend algorithms for prediction of heart failure related events in home care setting. Stud Health Technol Inform. 2017;236:219-26.

19. Ammenwerth E, Fetz B, Gstrein S, et al. Herzmobil, an integrated and collaborative telemonitoring-based disease management program for patients with heart failure: a feasibility study paving the way to routine care. JMIR Cardio. 2018;2(1):e11.

Publisher's Note Springer Nature remains neutral with regard to jurisdictional claims in published maps and institutional affiliations. 